La Méthode de Repère Mobile, La Théorie des Groupes Continus, et Les Espaces Généralisés. By Elie Cartan. (Actualités Scientifiques et Industrielles, No. 194.) Paris, Hermann, 1935. 65 pp.

In this booklet based on a series of lectures delivered in Moscow in 1930, Cartan develops some of the ideas of modern differential geometry from the point of view of ennuples of vectors. He begins with an examination of the method of the moving trihedral in classical differential geometry, observing that underlying this method are the two following conditions: 1) The trihedral, defined at each point of the manifold under consideration, must be determined intrinsically by the differential elements of the manifold, and 2) corresponding trihedrals of congruent curves (or surfaces) must be congruent. Several problems of classical differential geometry-minimal curves, homothetic curves, plane curves equivalent under the group of unimodular affine transformations, surfaces whose two fundamental forms have one linear factor in common-are considered in the light of his analysis of the method of the moving trihedral, and by the application of this method such topics as generalizations of the Frenet-Serret equations, curvature, arc, for these manifolds are developed.

The reader is thus led very naturally and easily to the more general problem-the application of these ideas to the fundamental principles of the theory of continuous groups. The way is quickly opened for a number of general theorems, Lie's constants of structure, and the Darboux-Maurer-Cartan equations of structure. The author investigates the role of these equations in a number of particular cases, and observes that "les équations de structure $d u$ groupe $G$ contiennent en elles toute la géométrie différentielle de l'espace doué du groupe fondamental $G, \ldots$. The last chapter is devoted to a brief, yet valuable, presentation of a number of questions in the theory of surfaces, Riemannian spaces, ruled spaces, and the more general linearly connected spaces.

This booklet, profusely illustrated with relatively simple examples, should be extremely valuable to all who wish to gain some insight into the meaning of the mass of generalizations that have been developed in this field in recent years. Unlike other works in this field, this one does not lose the reader in a maze of computations; yet the ideas are exhibited clearly and strikingly. The method of treatment employed serves to make this booklet unusually readable, while the emphasis on the geometric aspects not only adds to the lucidity, but serves to remind that modern differential geometry is geometry and not merely a branch of tensor analysis and differential equations.

HARRY LEVY

Intermediate Mechanics; Dynamics. By D. Humphrey. London, Longmans Green, 1930. xi+382 pp.

This is a good elementary text on analytical mechanics in the English tradition, offering a more explicit treatment of the elementary parts but not going as far as such standard texts as Jeans, Lamb, or Loney. Seven of the nine chapters are devoted to kinematics and dynamics of a particle in one or two dimensions, including the inevitable chapters on projectiles and simple harmonic motion; but two pages are given to motion under a central force, the reader being referred to more advanced books and advised that "the most important case is 
when $F$ is a gravitational force varying as the inverse square"! The book closes with two chapters on the most fundamental points in the dynamics of rigid bodies. The book might prove of value in a class of unusually gifted freshmen requiring more than the usual first term college mechanics, or for sophomores, but is, in the opinion of the reviewer, hardly advanced enough for upper class majors in physics or mathematics.

\section{H. P. Robertson}

La Causalité des Théories Mathématiques. By G. Bouligand. (Actualités Scientifiques et Industrielles, No. 184.) Paris, Hermann, 1934. 41 pp.

This booklet essays an investigation of the nature of generalizations by analysing the ultimate "causes" of mathematical proofs. The author distinguishes sharply between algorithmic proofs, which depend upon calculations, and direct proofs, which closely follow the intuition and so explain the real causes of the theorem. For example, the direct methods in the Calculus of Variations avoid artificial assumptions of differentiability. The same holds of the so-called "direct infinitesimal geometry"; in particular, Lebesgue's surface applicable to the plane but containing no line segment whatever is a striking revelation of the misleading results of algorithmic calculations.

A group of transformations can be viewed as a domain of causality. By this the author apparently means that the hypothesis and the conclusion of a geometric theorem should be invariant under the same group; if this is not the case the hypothesis or the conclusion can be generalized to give a more extended theorem. The numerous examples given include extensions of Meusnier's theorem and of the theorem of Dupin on triple orthogonal systems. This investigation of causes leads the author to argue for the objectivity of mathematics-that mathematics is a science studying a "mathematical nature" (the meaning of this naive phrase is not explained). The objective causes in this science are obtained not only by the discovery of proofs, but also in the formulation of axiom systems and in the discovery of significant concepts (for instance, the notion of semi-continuity). Such an emphasis on the importance of definitions is indeed refreshing.

Saunders MacLane

Matière et Énergie. By Victor Henri. Paris, Hermann, 1933. 436 pp.

This book is a solid and well-written text in modern physics. The introductory chapters take up the kinetic atomic theory of matter, $x$-ray spectra and atomic number, and the periodic system of the elements. These are followed by an account of natural and artificial radioactivity and the role of isotopes in the periodic system, with emphasis on the experimental conditions and findings; these chapters offer of course but a beginning in nuclear physics and must be supplemented by reports on later work. The last 200 pages present the elementary parts of kinetic theory and the Bohr theory of atomic structure, again with emphasis on the empirical findings. The book should be found very useful in obtaining a descriptive orientation in the complex fields touched upon, preparatory to a more detailed and more mathematical study; for such a purpose it can be highly recommended.

H. P. Robertson 